\title{
Singularidades da Convivência do Cônjuge e seu Parceiro Estomizado
}

\author{
Singularities of the Coexistence of Spouse and its Partner Ostomized
}

\author{
Singularidades de la Coexistencia del Esposo e su Socio Ostomizado
}

Ana Lucia da Silva', Ivone Kamada', João Batista de Sousa², André Luiz Vianna², Paulo Gonçalves de Oliveira²

\begin{abstract}
RESUMO
A convivência do cônjuge e seu parceiro estomizado exige adaptação do casal frente às mudanças impostas pela estomia intestinal. Objetivo: Descrever as singularidades da convivência marital do estomizado e seu cônjuge. Método: Trata-se de estudo descritivo, de natureza quantitativa, realizado com cônjuges de estomizados. Resultados: Participaram 36 cônjuges de estomizados, sendo $24(66,7 \%)$ do sexo feminino. A idade variou entre 31 a 70 anos. Quanto ao hábito de dormir dos cônjuges, 29 (80,6\%) dormiam com seu parceiro estomizado na mesma cama, sendo que 20 (55,6\%) cônjuges afirmaram que o estomizado nunca sujou a cama. No cotidiano, 25 (69,4\%) dos cônjuges sentiram odor desagradável exalado da estomia intestinal do seu parceiro, entretanto, 18 (50\%) não se incomodaram ao sentir odor exalado da estomia do seu parceiro. Das atividades sexuais, $11(30,6 \%)$ cessaram as relações sexuais, $25(69,4 \%)$ afirmaram que o saco coletor de fezes do cônjuge atrapalhava, 13 (36,1\%) tinham receio de machucar o parceiro estomizado e 13 (36,1\%) mudaram suas práticas sexuais em razão da presença da estomia do parceiro. Conclusão: A estomia intestinal permanente é um fato marcante no cotidiano do casal e limita as atividades sexuais. Os cônjuges mudaram suas práticas sexuais ou cessaram as relações íntimas após a confecção do estoma do parceiro, apesar de serem relativamente jovens.
\end{abstract}

DESCRITORES: Estomaterapia. Cônjuges. Estomia. Sexualidade.

\begin{abstract}
The coexistence of the spouse and your partner ostomyzed requires adaptation of the couple to the changes imposed by the intestinal ostomy. Objective: To describe the singularities of the marital coexistence of ostomyzed patients and their spouses. Method: This is a descriptive quantitative study, conducted with ostomyzed spouses. Results: Participants were 36 spouses of ostomy, 24 (66.7\%) were female. The age ranged from 31 to 70 years. Regarding the habit of sleeping spouse, 29 (80.6\%) slept with your partner ostomyzed in the same bed, and 20 (55.6\%) stated that the spouses ostomy never messed the bed. In daily life, 25 (69.4\%) of the spouses felt unpleasant odor exhaled from their partners, however, 18 (50\%) did not bother to feel the odor of the partners' ostomy. About the sexual activity, 11 (30.6\%) ceased sex, 25 (69.4\%) said the feces collector bag of the spouses disrupts, 13 (36.1\%) are afraid of hurting the ostomyzed partner and $13(36.1 \%)$ changed their sexual practices because of the partner's stoma. Conclusion: The intestinal ostomy permanent is a remarkable fact in the couple's daily life and limits the sexual activities. Spouses have changed their sexual practices or ceased intimate relations after confection of partner ostomy, despite being relatively young.
\end{abstract}

DESCRIPTORS: Stomatherapy. Spouses. Ostomy. Sexuality.

'Departamento de Enfermagem da Faculdade de Ciências da Saúde da Universidade de Brasília (UnB) - Brasília (DF), Brasil.

Endereço para correspondência: Departamento de Enfermagem, Faculdade de Ciências da Saúde, Universidade de Brasília, Câmpus Darcy Ribeiro CEP: 70910-900 - Asa Norte - Brasília (DF), Brasil - E-mail: analucia@unb.br

2Departamento de Cirurgia da Faculdade de Medicina da UnB - Braśília (DF), Brasil.

Artigo recebido em: 22/04/2015 - Aceito para publicação em: 15/06/2015 


\section{RESUMEN}

La coexistencia del cónyuge y su socio ostomía requiere una adaptación de la pareja a los cambios impuestos por la ostomía. Objetivo: Describir las singularidades de la convivencia matrimonial de los pacientes ostomizados y su cónyuge. Método: Se trata de un estudio descriptivo, cuantitativo, realizado con cónyuges del ostomízados. Resultados: Los participantes fueron 36 los cónyuges de ostomizados, 24 (66,7\%) eran mujeres. El rango de edad fue 31 a 70 años. En cuanto a la costumbre de dormir cónyuge, 29 (80,6\%) descansan con tu pareja ostomía en la misma cama, y 20 (55,6\%) afirmaron que la ostomía del cónyuges nunca mal estado de la cama. En la vida diaria, 25 (69,4\%) de los cónyuges se sintieron exhala olor desagradable de ostomía su pareja, sin embargo, 18 (50\%) no se molestó a sentir olor exhalado de ostomía su pareja. La actividad sexual, 11 (30,6\%) dejó de sexo, 25 (69,4\%) declaró que el esposo de la bolsa dificulta colector de heces, 13 (36,1\%) tienen miedo de lastimar al compañero de ostomía y 13 (36,1\%) cambió sus prácticas sexuales debido estoma de la pareja. Conclusión: Permanente ostomía es un hecho notable en la vida cotidiana de la pareja y limita las actividades sexuales. Los esposos han cambiado sus prácticas sexuales o ha dejado las relaciones íntimas después de hacer estoma pareja, a pesar de ser relativamente joven.

DESCRIPTORES: Estomaterapia. Esposos. Estomía. Sexualidad.

\section{INTRODUÇÃO}

A estomia intestinal é uma situação que atinge pessoas de ambos os sexos. Estas são afetadas de formas diferentes após a confecção do estoma. O descontrole da expulsão dos gases e do conteúdo fecal pela estomia é o que mais atemoriza a pessoa estomizada. Esta experimenta sentimentos de não ser capaz de levar uma vida normal. A saída dos gases provoca barulho e deixa todos constrangidos, tanto o estomizado como os que estão à sua volta, pois se trata de ato incomum publicamente. $\mathrm{O}$ impacto gerado por uma estomia intestinal permanente abrange a percepção da alteração da imagem corporal, da sexualidade e do modo de vida que comprometem a qualidade de vida.

Estudos têm mostrado que problemas psicossociais, sexuais e isolamento social são questões centrais para a pessoa com estomia intestinal e podem permanecer mesmo após a sua adaptação à nova situação ${ }^{1,2}$. Nesse sentido, as pessoas estomizadas ficam com inúmeras dúvidas e desconhecem cuidados importantes para a manutenção de uma vida saudável, mesmo após um tempo relativamente longo da realização da cirurgia ${ }^{2}$.

$\mathrm{Na}$ avaliação da qualidade de vida de pacientes iranianos com colostomia permanente foi detectado que, após a estomia intestinal, a função sexual de homens e mulheres ficou prejudicada ${ }^{3}$.

Dessa forma, acredita-se que a estomia intestinal permanente gera alterações tão relevantes para o estomizado afetando também os cônjuges o que pode influenciar na convivência diária do casal. Silva et al. ${ }^{4}$ concluíram que ambas as partes da relação cônjuge e parceiro estomizado sofrem alterações no âmbito da sexualidade. Nesse sentido, exige-se adaptação do casal às mudanças decorrentes da estomia, pois são permeadas de sentimentos conflituosos que atingem os aspectos fisiológicos, psíquicos, sociais e sexuais.

Estudo que avaliou cônjuges de pacientes com câncer mostrou que houve aumento nos custos com saúde, inclusive com aumento de diagnósticos de doenças psiquiátricas entre os cônjuges, especialmente para aqueles de pacientes com câncer de cólon e de pulmão ${ }^{5}$.

Assim, é preciso dar mais atenção ao cônjuge, considerando que este representa fonte de apoio emocional, suporte e o propósito na vida do parceiro, que são considerados os principais recursos que promovem o bem-estar físico do indivíduo ${ }^{6}$. A convivência marital considerada estável tem impactos positivos para a satisfação pessoal. Em contrapartida, uma relação conflituosa influi negativamente na dinâmica familiar, no emocional e no comportamento dos parceiros ${ }^{6}$. $\mathrm{O}$ cônjuge é, muitas vezes, o parente mais próximo do doente; por isso, sua resposta emocional tem um papel relevante na adaptação do seu parceiro estomizado.

Em estudo de revisão sistemática foi detectado que os cônjuges de estomizados têm problemas psicossociais significativos e que atualmente não são tratados na prática clínica diária. Além disso, quando o estomizado apresenta sintomas de depressão, o cônjuge também sofre com ele. Os cônjuges se preocupam com questões relativas ao funcionamento sexual e social e sentem necessidade de mais apoio para se adaptar à nova situação ${ }^{7}$. Viver com uma estomia definitiva afeta negativamente não apenas o paciente, 
mas também o cônjuge nos aspectos físico, social, psicológico e no estilo de vida ${ }^{8}$.

O presente estudo enfocou a percepção do cônjuge acerca da convivência diária após a estomia intestinal permanente do parceiro. Como há pouca informação disponível na literatura médica sobre o cotidiano do cônjuge e seu parceiro estomizado, os pesquisadores destacam a necessidade do planejamento do cuidado a esse casal, embasado não só no conhecimento científico, mas também na caracterização da realidade, percepções e vivências.

Considerando a importância do tema, este o estudo teve por objetivo descrever as singularidades da convivência do cônjuge e seu parceiro estomizado bem como a atividade sexual e a presença do estoma.

\section{MÉTODO}

Trata-se de estudo descritivo, de natureza quantitativa, realizado com cônjuges de estomizados. O projeto foi aprovado pelo Comitê de Ética em Pesquisa em Seres Humanos da Faculdade de Medicina da Universidade de Brasília (UnB), sob o número de protocolo (CEP-FM 011/2009) e todos os participantes assinaram o Termo de Consentimento Livre e Esclarecido.

A população teve por base os cadastros de dados secundários do Núcleo de Atenção Básica e do Estomizado da Secretaria de Estado de Saúde do Distrito Federal. Em outubro de 2011, existiam cadastrados entre crianças e adultos no Programa de Assistência Ambulatorial 685 pacientes com estomia.

Neste estudo, foram denominados cônjuges os parceiros ou companheiros, independentemente do estado civil. E como critérios de inclusão foram considerados os seguintes aspectos: aceitar participar do estudo; ter idade igual ou superior a 18 anos; ser alfabetizado; possuir fluência falada e escrita em língua portuguesa; ser cônjuge de pessoa com estomia intestinal e ter convivido, no mínimo um ano antes e um ano após a confecção da estomia. Outro aspecto considerado foi o de que os parceiros estomizados fossem portadores de estomia intestinal de caráter permanente.

A coleta dos dados foi realizada no período de outubro de 2011 a junho de 2012 por meio de questionário composto por três partes: dados sociodemográficos, aspectos inerentes a estomia e aspectos sexuais. As questões foram do tipo múltipla escolha, ou opções sim ou não. O questionário foi respondido individualmente, sem a presença de quaisquer outras pessoas, ficando o pesquisador em ambiente próximo caso houvesse necessidade de algum esclarecimento.

As variáveis de interesse relacionadas à estomia intestinal foram utilizadas para avaliar as reações comportamentais do cônjuge acerca do hábito de dormir, do odor exalado da estomia do seu parceiro, da ocorrência de situações embaraçosas e da atividade sexual.

Foi elaborado instrumento específico para o presente estudo. O questionário foi construído pelos autores, a partir da experiência, de mais de 20 anos, no atendimento à pessoas estomizadas. Em seguida, os questionários foram revisados por cinco especialistas, pesquisadores nas áreas do conhecimento metodológico, linguístico e da saúde. Os instrumentos foram, inicialmente, aplicados a cinco cônjuges com o objetivo de avaliar a adequação das questões, se eram suficientemente claras para que pudessem ser respondidas com segurança e sem margem para duplo entendimento. Após análise prévia, foi composto o instrumento aplicado na pesquisa.

Os resultados foram organizados em tabelas e analisados com o auxílio do programa Statistical Package for Social Sciences (SPSS) - 20.0 for Windows, por meio de análise estatística descritiva, expressos como frequências absolutas e relativas (percentagens).

\section{RESULTADOS}

A amostra final do estudo foi composta por 36 cônjuges, de pessoa com estomia intestinal permanente indicada por câncer do reto distal. O quantitativo de participantes expresso é decorrente do atendimento aos critérios estabelecidos, e com isso, reduzido consideravelmente.

Quanto ao sexo, faixa etária, grau de instrução, tempo de união e renda familiar, os dados estão descritos na Tabela 1.

Quanto ao hábito de dormir dos cônjuges dos estomizados, episódios de extravasamento de fezes da estomia e sujar a cama do casal enquanto dormiam, às reações comportamentais dos cônjuges diante da situação de extravasamento de fezes da estomia do parceiro no leito do casal, à percepção de odor desagradável exalado da estomia intestinal do seu parceiro no cotidiano e à noite na intimidade do casal e, referente às reações mediante à percepção de odor desagradável, os dados estão descritos na Tabela 2. Neste estudo, a ocorrência de situações constrangedoras relacionadas à 
estomia envolvendo o casal foi analisada e constatou-se que 20 (55,6\%) dos cônjuges vivenciaram situações embaraçosas.

Das atividades sexuais relacionadas ao uso do equipamento coletor, receio de machucar o parceiro estomizado e mudanças na prática sexual dos cônjuges entrevistados, os dados estão descritos na Tabela 3.

\section{DISCUSSÃO}

A identificação das particularidades da convivência marital do cônjuge e do parceiro com estomia intestinal permanente é o ponto de partida deste estudo. Com relação à idade dos entrevistados, foi observado que a maioria (72,3\%) encontrava-se na faixa etária de 31 a 60 anos. Entretanto, vale destacar que 44,5\% dos cônjuges encontravam-se abaixo de 50 anos. Em relação ao sexo, mostrou que grande parte do grupo investigado era do sexo feminino.

Tabela 1. Distribuição de cônjuges de estomizados segundo dados de sexo, faixa etária, grau de instrução, tempo de união e renda familiar $(n=36)$, Brasília (DF), Brasil, 2012.

\begin{tabular}{|c|c|c|}
\hline & $\mathrm{n}$ & $\%$ \\
\hline \multicolumn{3}{|l|}{ Sexo } \\
\hline Feminino & 24 & 66,7 \\
\hline Masculino & 12 & 33,3 \\
\hline \multicolumn{3}{|l|}{ Faixa etária } \\
\hline 31 a 40 anos & 5 & 13,9 \\
\hline 41 a 50 anos & 11 & 30,6 \\
\hline 51 a 60 anos & 10 & 27,8 \\
\hline 61 a 70 anos & 6 & 16,6 \\
\hline Acima de 70 anos & 4 & 11,1 \\
\hline \multicolumn{3}{|l|}{ Grau de instrução } \\
\hline Ensino fundamental incompleto & 4 & 11,1 \\
\hline Ensino fundamental completo & 8 & 22,2 \\
\hline Ensino médio incompleto & 2 & 5,6 \\
\hline Ensino médio completo & 8 & 22,2 \\
\hline Ensino superior & 14 & 38,8 \\
\hline \multicolumn{3}{|l|}{ Tempo de união } \\
\hline Menos de 2 anos & 1 & 2,8 \\
\hline De 2 a 5 anos & 0 & 0,0 \\
\hline Mais de 5 anos & 35 & 97,2 \\
\hline \multicolumn{3}{|l|}{ Renda familiar } \\
\hline Até 1 salário mínimo & 1 & 2,8 \\
\hline Mais de 1 até 2 salários mínimos & 10 & 27,8 \\
\hline Mais de 2 até 5 salários mínimos & 6 & 16,6 \\
\hline Mais de 5 salários mínimos & 19 & 52,8 \\
\hline Total & 36 & 100,0 \\
\hline
\end{tabular}

Foi observado que a maioria dos cônjuges incluídos neste estudo tinha grau de escolaridade superior. Esses resultados foram consistentes com estudo que avaliou a qualidade de vida dos colostomizados no Irã e concluíra que os

Tabela 2. Cotidiano do cônjuge e seu parceiro estomizado ( $n=36)$, Brasília (DF), 2012.

\begin{tabular}{|c|c|c|}
\hline Variáveis & $\mathrm{n}$ & $\%$ \\
\hline \multicolumn{3}{|c|}{$\begin{array}{l}\text { Dormir em cama separada depois que o parceiro } \\
\text { ficou estomizado }\end{array}$} \\
\hline Não & 29 & 80,6 \\
\hline $\mathrm{Sim}$ & 7 & 19,4 \\
\hline \multicolumn{3}{|c|}{$\begin{array}{l}\text { Sujar a cama do casal com fezes da estomia } \\
\text { enquanto dormiam }\end{array}$} \\
\hline Não & 20 & 55,6 \\
\hline $\mathrm{Sim}$ & 16 & 44,4 \\
\hline \multicolumn{3}{|c|}{$\begin{array}{l}\text { Comportamento dos cônjuges frente à situação } \\
\text { de perda de fezes na cama }\end{array}$} \\
\hline Não se sentiam incomodados & 23 & 63,9 \\
\hline odados & 10 & 27,8 \\
\hline Não responderam a questão & 3 & 8,3 \\
\hline \multicolumn{3}{|c|}{ Sentir odor de fezes à noite na intimidade do casal } \\
\hline Não & 12 & 33,3 \\
\hline $\operatorname{Sim}$ & 24 & 66,7 \\
\hline \multicolumn{3}{|l|}{ Sentir odor de fezes no cotidiano } \\
\hline Não & 11 & 30,6 \\
\hline $\mathrm{Sim}$ & 25 & 69,4 \\
\hline
\end{tabular}

Comportamento dos cônjuges frente à situação do parceiro exalar odores desagradáveis da estomia

\begin{tabular}{lll} 
Não emitiram opinião & 2 & 5,6 \\
\hline Incomodam & 16 & 44,4 \\
Não incomodam & 18 & 50,0 \\
\hline
\end{tabular}

Tabela 3. Atividade sexual dos cônjuges e a presença da estomia ( $n=36)$, Brasília (DF), 2012.

\begin{tabular}{|c|c|c|}
\hline Aspectos sexuais & $\mathrm{n}$ & $\%$ \\
\hline \multicolumn{3}{|c|}{ Atividade sexual e o uso do equipamento coletor } \\
\hline Atrapalha & 12 & 33,3 \\
\hline Não atrapalha & 13 & 36,1 \\
\hline Ausência de relações sexuais & 11 & 30,6 \\
\hline \multicolumn{3}{|c|}{$\begin{array}{l}\text { Receio de machucar o parceiro estomizado durante } \\
\text { as relações sexuais }\end{array}$} \\
\hline $\operatorname{Sim}$ & 13 & 36,1 \\
\hline Não & 12 & 33,3 \\
\hline Ausência de relações sexuais & 11 & 30,6 \\
\hline \multicolumn{3}{|c|}{$\begin{array}{l}\text { Mudanças nas práticas sexuais advindas da } \\
\text { estomia do parceiro }\end{array}$} \\
\hline $\operatorname{Sim}$ & 13 & 36,1 \\
\hline Não & 9 & 25,0 \\
\hline Sem resposta & 14 & 38,9 \\
\hline
\end{tabular}


participantes tinham um alto nível educacional, fato que pode influenciar nas atitudes dos pacientes em relação à estomia e os mecanismos adaptativos e, assim, facilitar a convivência com o cônjuge ${ }^{3}$. Quanto mais elevado os níveis de conhecimento acerca dos cuidados com o estoma, melhor adaptação psicossocial'.

Quanto ao tempo de união dos cônjuges, a maioria tinha mais de cinco anos de união estável. Isso é justificável considerando-se o tempo de união como critério de inclusão deste estudo (mínimo de um ano antes da confecção da estomia do seu parceiro e um ano após). Os entrevistados afirmaram que seus parceiros estomizados possuem estomia intestinal permanente há mais de cinco anos.

Destaca-se que a pessoa que está em união estável e fica estomizada, geralmente, conta com o apoio do cônjuge. Entretanto, os que não tinham um relacionamento sólido inclinaram-se a ficar sozinhos após a confecção da esto$\mathrm{mia}^{1}$. A renda familiar média ficou acima de cinco salários mínimos para a maioria dos entrevistados, o que representa cerca de 20.000 dólares anuais.

Em relação ao hábito de dormir dos cônjuges, observou-se que a maioria $(80,6 \%)$ deles dorme junto com seus parceiros, na mesma cama, não havendo modificação deste hábito. No entanto, verificou-se que quase $20 \%$ dos cônjuges dormiam em camas separadas, quando foram analisados os que sempre ou eventualmente tinham esse hábito.

$\mathrm{Na}$ avaliação do evento sujar a cama do casal com fezes da estomia enquanto dormia, a maioria dos cônjuges revelou que não presenciou esses episódios. Entretanto, vale destacar a percentagem próxima dos $44,4 \%$ que presenciaram a ocorrência desses eventos. Quanto às reações dos cônjuges de estomizado mediante este fato, o que chama a atenção é a forma com que aceitam esse fato, pois $63,9 \%$ responderam que não se sentiram incomodados, o que denota certa passividade. Contudo, vê-se que 27,8\% dos cônjuges alegaram que se sentiram incomodados e outros que preferiram não responder esta questão, optando pelo silêncio.

Neste estudo, a percepção de odor desagradável da estomia intestinal do parceiro foi avaliada em dois momentos diferentes; no período noturno, na intimidade do casal, e no cotidiano do cônjuge. Foi constatado que no período noturno a maioria dos cônjuges $(66,7 \%)$ declarou que sentiu odores de fezes à noite, na intimidade do casal. Mas ressalta-se que um terço deles nunca sentiu odores de fezes à noite nos aposentos do casal. Quando a avaliação foi do seu cotidiano, a maioria $(69,4 \%)$ dos cônjuges sentiu odores desagradáveis exalados da estomia intestinal do seu parceiro. Esse é um resultado que justifica a preocupação da pessoa com estomia intestinal e é condizente com a literatura nacional e internacional. A literatura traz relatos dos estomizados acerca do incômodo da eliminação involuntária de gases, odor de fezes exalado e extravasamentos de fezes pela estomia ${ }^{8,9}$.

A perda do controle da eliminação de fezes e gases representa grandes desafios ao estomizado e ao cônjuge, ocasionando, inevitavelmente, a ocorrência de situações constrangedoras. A expulsão de gases, que geralmente emite ruídos sonoros, constitui importante causa de constrangimento ${ }^{10}$. Para os cônjuges que cuidam do seu parceiro, a eliminação de gases, o seu ruído e odor exalado pela estomia são fatores que os incomodam ${ }^{8}$. Atualmente, novos equipamentos adjuvantes de proteção e segurança aos estomizados, desenvolvidos com os avanços na tecnologia, podem diminuir os problemas relacionados ao vazamento de fezes, odores e gases que interferem nas atividades de vida diária da pessoa com estomia intestinal.

Quanto às reações apresentadas, constatou-se que apesar da percepção de odor desagradável da estomia do seu parceiro, metade dos cônjuges entrevistados não se aborreceu com esse fato, porém outros $44,4 \%$ sentiram desconfortáveis e incomodados com a situação. Vale destacar que houve aqueles que não responderam ao questionamento.

Neste estudo, em relação à ocorrência de situações embaraçosas relacionadas à estomia envolvendo o casal, constatou-se que 55,6\% dos parceiros vivenciaram situações incômodas. O receio de ocorrer vazamento de fezes fora do coletor, a emissão de ruídos e gases com odores desagradáveis são as principais causas de situações constrangedoras entre as mulheres jovens com estomia intestinal ${ }^{3}$. O tempo é fator relevante no ajustamento à nova condição ${ }^{11}$. Geralmente, as situações embaraçosas relacionadas à estomia intestinal ocorrem ao longo dos anos, após a confecção do estoma e há necessidade de constante adaptação ao novo estilo de vida.

As questões relacionadas à sexualidade são difíceis de serem avaliadas por se tratar de um tema subjetivo, que abrange os aspectos associados às modificações advindas da estomia. Em nosso estudo, 38,9\% dos participantes deixaram sem respostas os questionamentos sobre as mudanças na atividade sexual. Assim, os resultados aqui obtidos são semelhantes aos de outros autores que avaliaram a qualidade de vida dos estomizados tailandeses. Esse trabalho mostrou que a maior parte das questões relativas à atividade sexual não foram respondidas, o que foi atribuído à 
atitudes conservadoras dos sujeitos da pesquisa em discutir esse tipo de assunto ${ }^{12}$. Na literatura, existem evidências de que a estomia intestinal afeta as atividades sexuais. Isso pode estar associado ao elevado nível de comprometimento emocional e sexual em homens e mulheres com colostomia, sendo mais acentuado nas mulheres ${ }^{3,13}$.

Em relação aos dados referentes à presença do equipamento coletor durante a relação sexual, a maioria dos cônjuges não identificou como fator complicador. No entanto, 33,3\% dos cônjuges referiram como obstáculo na relação sexual com o estomizado. $O$ fato é que a estomia e o equipamento coletor imprimem mudanças concretas e materializam a realidade de ser estomizado. $\mathrm{O}$ contato e a pressão exercida no equipamento coletor podem inibir os movimentos do ato sexual e ocasionar perda de interesse do cônjuge. Apesar do progresso da tecnologia na criação de equipamentos coletores, existe o receio de perdas acidentais de gases ou fezes, especialmente em situações de atrito durante $\mathrm{o}$ ato sexual ${ }^{14}$.

Ainda há que se considerar a estomia e o saco coletor das fezes como obstáculo na aparência física, que deixam uma sensação diminuída de beleza e higiene o que pode contribuir para uma relação sexual insatisfatória. Além disso, há possibilidade de ruptura durante o ato sexual o que pode culminar em situação constrangedora ao casal ${ }^{15}$. Estudo comparativo realizado com cônjuges de estomizado e cônjuges de não estomizado, aponta reação negativa nas atividades sexuais e desempenho sexual insatisfatório do parceiro estomizado ${ }^{4}$.

Quanto ao receio de machucar o estomizado durante o ato sexual, foi constatado percentagens próximas entre os que se preocupavam e os que não se preocupavam com essa possibilidade. Divergente desses resultados, alguns estudos concluíram que muitos cônjuges reagiram negativamente, ou com cautela, por medo de prejudicar o estoma do parceiro. Essa reação pode ocasionar diminuição ou cessação da atividade sexual ${ }^{14,15}$.

Em relação às mudanças nas práticas sexuais dos cônjuges devido à presença da estomia, apenas $25 \%$ dos cônjuges não mudaram suas práticas sexuais. Considerando os que não responderam a esse questionamento e os que declararam mudanças, é possível afirmar que a estomia intestinal do companheiro provocou mudanças nas práticas sexuais dos cônjuges. Nesse ponto, o número de cônjuges que não respondeu a esse questionamento foi maior do que o habitual apresentado anteriormente, ou seja, $38,8 \%$ dos cônjuges deixaram sem resposta a avaliação da presença da estomia nas práticas sexuais do casal. Contudo, essa informação é coerente com resultados já apresentados, em que um número relevante de cônjuges não tem relação sexual. Outra pesquisa também constatou que as pessoas com estomia intestinal manifestaram insegurança quanto a sua sexualidade, o que pode ter ocasionado mudanças em suas práticas sexuais ${ }^{14}$.

Em estudo que avaliou a experiência de mulheres com estomia intestinal, conclui-se que algumas delas não tinham a relação sexual como ponto central da sua vida conjugal, e que a falta de relações sexuais não era tão angustiante e optaram por outros atos de intimidade no relacionamento do casal ${ }^{16}$. A atividade sexual após a estomia pode ser considerada como algo não essencial na vida dos estomizados, pois sentem muita vergonha e geralmente abdicam do ato sexual, buscando outros tipos de compensação ${ }^{1}$.

No presente estudo considerou-se que houve mudanças significativas nas práticas sexuais dos cônjuges, uma vez que uma parte representativa deixou de ter práticas sexuais devido às mutilações após a cirurgia do seu parceiro. É relevante o número daqueles que cessaram as atividades sexuais, ou seja, não tem mais relação sexual com seu parceiro.

Nesse sentido, estudo que avaliou 26 homens e 30 mulheres demonstrou que, mesmo sendo sexualmente ativos antes de seus parceiros serem colostomizados, 20 homens e 10 mulheres estavam inativos depois da estomia intestinal do parceiro. Dos homens, 16 (80\%) relataram que a razão para ter cessado suas atividades sexuais era o sentimento de repulsa pela colostomia de suas esposas ${ }^{8}$.

Embora não tenha sido objeto do presente estudo a exploração de facetas subjetivas das atividades sexuais dos entrevistados, verificou-se que os dados relativos às mudanças nas práticas sexuais são condizentes com estudo anterior, que afirma serem tão profundas as modificações ocorridas para as pessoas estomizadas que o ato sexual torna-se secundário, podendo ser substituído por sentimentos como amor, carinho, respeito, companheirismo e outros ${ }^{1}$. Nos casos em que havia um relacionamento estável antes da estomia, as dificuldades foram gradativamente trabalhadas pelos cônjuges na busca conjunta de soluções adequadas para ambos ${ }^{14}$.

Estudos anteriores ressaltam que a mulher estomizada teve maior perda da função sexual do que o homem estomi$\mathrm{zado}^{3,16}$. No entanto, em nosso estudo, a maioria entrevistada foram mulheres, logo, estavam representando seus parceiros homens estomizados, o que permite inferir que as mudanças 
na prática sexual são originadas por razões diversas e atingem não só o estomizado, mas também o seu cônjuge.

Estudo realizado em uma sociedade iraniana aponta que os estomizados casados apresentaram alguns problemas sexuais no início da doença ou após a cirurgia, e que se resolveram esses problemas gradativamente após discussão com seus pares ${ }^{11}$.

É compreensível que a estomia intestinal influencie a sexualidade devido à falta de controle das eliminações fecais e gases, do barulho provocado, como também do equipamento coletor de uso contínuo e visualmente não atraente ${ }^{16}$. Com a autoimagem alterada, estomizado e cônjuge adotam mecanismos de defesa, pois, há que se considerar aquele que participa efetivamente do relacionamento pode ver assolado por sentimentos conflitantes de desejo e, ao mesmo tempo, repulsa, nojo e compaixão ${ }^{17}$.

Esse tipo de problema ilustra a necessidade de novas estratégias de assistência ao estomizado e seu cônjuge. Portanto, é preciso dar mais atenção para o universo do cônjuge da pessoa com estomia intestinal permanente, buscando conhecê-lo e compreendê-lo na sua temporalidade, mediante a interpretação da sua percepção.

Dessa forma, os resultados referentes à vivência do cônjuge e seu parceiro estomizado devem ser analisados com cautela por tratar-se de um tema subjetivo e sujeito à imprecisão nas respostas. Contudo, acredita-se que esse estudo da convivência conjugal com a pessoa estomizada possa contribuir para maior entendimento dos aspectos sociais, psíquicos e sexuais do cotidiano desses cônjuges.

\section{Limitações}

No presente estudo, consideram-se como fator de limitação as singularidades da convivência marital por tratar-se de questões de foro íntimo e as informações prestadas podem ter valores diferentes dos reais. Ainda há que se destacar o aspecto da sexualidade dos casais, que não foi avaliado antes da operação que resultou na estomia intestinal. $\mathrm{O}$ reconhecimento de outros fatores pode estar associado ao comportamento do parceiro na adaptação psicossocial. Dessa forma, torna-se necessário a realização de mais pesquisas que possam elucidar e ajudar os cônjuges e seus parceiros a lidar melhor com a estomia intestinal.

\section{CONCLUSÃO}

A sexualidade é um tema difícil de ser avaliado por tratar-se de questões de foro íntimo e as respostas podem não retratarem fielmente o julgamento da consciência das pessoas acerca das situações de sua intimidade.

Entretanto, o presente estudo evidencia que os cônjuges de pessoas com estomia intestinal permanente, possivelmente, sofrem um impacto e são afetados negativamente no seu cotidiano. Essas constatações permitem afirmar que a estomia influencia nas atividades sociais e sexuais dos casais, pois é um fator limitador, principalmente da prática sexual, uma vez que um número significativo de cônjuges participantes deste estudo cessaram as atividades sexuais, apesar de serem relativamente jovens.

Apesar das circunstâncias negativas que envolvem a convivência marital, o casal procura partilhar estratégias no sentido de adaptar à vida, com a estomia, e manter a harmonia da união.

Assim, espera-se que os resultados apresentados possam fortalecer uma assistência multidisciplinar centrada no cônjuge e seu parceiro com estomia intestinal permanente, e contribuir para desmistificar e esclarecer as questões acerca dos aspectos sociais e sexuais do cônjuge e seu parceiro estomizado.

\section{REFERÊNCIAS}

1. Silva AL, Shimizu HE. O significado da mudança no modo de vida da pessoa com estomia intestinal definitiva. Rev Lat Am Enfermagem. 2006;14(4):483-90.

2. Poletto D, Silva DMGV. Viver com estoma intestinal: a construção da autonomia para o cuidado. Rev Lat Am. Enfermagem. 2013;21(2):[08 telas].

3. Mahjoubi B, Mirzaei R, Azizi R, Jafarinia M, Zahedi-Shoolami L. A cross-sectional survey of quality of life in colostomates: a report from Iran. Health Qual Life Outcomes. 2012;10:136.
4. Silva AL, Monteiro PS, Sousa JB, Vianna AL, Oliveira PG. Partners of patients having a permanent colostomy should also receive attention from the healthcare team. Colorectal Dis. 2014;16(12):431-4.

5. Sjövall K, Attner B, Lithman T, Noreen D, Gunnars B, Thome $B$, et al. Influence on the health of the partner affected by tumor disease in the wife or husband based on a populationbased register study of cancer in Sweden. J Clin Oncol. 2009; 27(28):4781-6. 
6. Silva TBC, Santos MCL, Almeida AM, Fernandes AFC. Percepção dos cônjuges de mulheres mastectomizadas com relação à convivência pós-cirurgia. Rev Esc Enferm USP. 2010;44(1):113-9.

7. Danielsen AK, Burcharth J, Rosenberg J. Spouses of patients with a stoma lack information and support and are restricted in their social and sexual life: a systematic review. Int J Colorectal Dis. 2013;28(12):1603-12.

8. Cakmak A, Aylaz G, Kuzu MA. Permanent stoma not only affects patients' quality of life but also that of their spouses. World J Surg Oncol. 2010;34(12):2872-6.

9. Cheng F, Meng A, Yang L, Zhang Y. The Correlation Between Ostomy Knowledge and Self-Care Ability with PsychosocialAdjustment in Chinese Patients with a Permanent Colostomy: A Descriptive Study. Ostomy Wound Management. 2013;59(7):35-8.

10. Mirand SM, Nascimento CMFS, Luz MHBA, Andrade EMLR, Luz ALA, Torres CRD. Viver com Estomia: Contribuições para a Assistência de Enfermagem. Rev Estima. 2014;12 (3):33-40.
11. Dabirian A, Yaghmaei F, Rassouli M, Tafreshi MZ. Quality of life in ostomy patients: a qualitative study. Patient Prefer Adherence. 2011;5:1-5.

12. Maneesin S, Sampatanukul P, Lertmaharit S, Na Nagara C, Prasopsanti K. Health-related quality of life of patients living with ostomy in Thailand and cost implications. Asian Biomedicine. 2012;6(2):235-43.

13. Kiliç E, Taycan O, Belli AK, Özmen M. The effect of permanent ostomy on body image, self-esteem, marital adjustment, and sexual functioningg. Turk Psikiyatri Derg. 2007;18(4):1-8.

14. Paula MAB, Takahashi RF, Paula PR. Experiencing sexuality after intestinal stoma. J Coloproctol. 2012;32(2):163-74.

15. Sprunk $E$, Alteneder RR. The impact of an ostomy on sexuality. Clin J Oncol nurs. 2000;4(2):85-8.

16. Ramirez M, McMullen C, Grant M, Altschuler A, Hornbrook Mc, Krouse RS. Figuring out sex in a reconfigured body: experiences of female colorectal cancer survivors with ostomies. Women Health. 2009;49(8):608-24.

17. Barbutti RCS, Silva MCP, Abreu MAL. Ostomia, uma difícil adaptação. Rev SBPH. 2008;11(2):27-39. 